\title{
Nonmonotonic Field Dependence of Damping and Reappearance of Rabi Oscillations in Quantum Dots
}

\author{
A. Vagov, ${ }^{1,2}$ M. D. Croitoru, ${ }^{3}$ V. M. Axt, ${ }^{4}$ T. Kuhn, ${ }^{4}$ and F. M. Peeters ${ }^{2}$ \\ ${ }^{1}$ Physics Department, Lancaster University, Lancaster, United Kingdom \\ ${ }^{2}$ CMT, Departement Fysica, Universiteit Antwerpen, Antwerpen, Belgium \\ ${ }^{3}$ EMAT, Departement Fysica, Universiteit Antwerpen, Antwerpen, Belgium \\ ${ }^{4}$ Institut für Festkörpertheorie, Westfälische Wilhelms-Universität Münster, Germany
}

(Received 15 May 2006; published 1 June 2007)

\begin{abstract}
The dynamics of strongly confined laser driven semiconductor quantum dots coupled to phonons is studied theoretically by calculating the time evolution of the reduced density matrix using a numerical path integral method. We explore the cases of long pulses, strong dot-phonon and dot-laser coupling, and high temperatures, which, up to now, have been inaccessible. We find that the phonon-induced damping of Rabi rotations is a nonmonotonic function of the laser field that is increasing at low fields and decreasing at high fields. This results in a reappearance of Rabi rotations at high fields. This phenomenon is of a general nature which occurs for all temperatures and carrier-phonon coupling strengths.
\end{abstract}

DOI: 10.1103/PhysRevLett.98.227403

The controlled manipulation of localized excitonic states in semiconductor nanosize quantum dots (QD) by using Rabi oscillations (RO) is a key ingredient in many proposals for quantum information applications in a solid state environment [1]. Typically RO in QDs are measured by recording the rotation of the Bloch vector after a pulse with given duration as a function of the pulse area (PA) [25]. We shall refer to these signals as Rabi rotations (RR) to distinguish them from the temporal evolution during the pulse which we will call RO. In real systems the coherence of the carrier states is destroyed leading to a damping of RO which reflects itself in a damping of the observed RR. This damping may be caused by different mechanisms. Interface dots, e.g., typically exhibit a strong radiative decay due to large dipole couplings $[2,3]$. Tunneling induces dephasing when RR are measured in the photocurrent $[4,6]$. Also off-resonant wetting layer states may contribute to the decay $[5,6]$. The coupling of phonons to the QD provides a basic dephasing mechanism present in any sample and all setups and thus marks a lower limit for the decoherence [7-9]. In typical self-assembled QDs it is indeed the elastic phonon scattering, usually referred to as pure dephasing, which dominates the loss of coherence on a picosecond time scale at temperatures below $\sim 100 \mathrm{~K}$ $[10,11]$.

The most widely studied model for phonon-induced pure dephasing in strongly confined QDs accounts for two electronic levels coupled to a laser field and to noninteracting phonons $[8-10,12]$. This two-level independent Boson model (TLIB) is a special case of a spin Boson model. Such models often serve as prototypes for quantum dissipative systems and thus have wide applications in many areas of physics [13]. Quantum dissipation as described by the TLIB reveals the genuine non-Markovian nature of pure dephasing processes which makes these systems attractive for studies of non-Markovian dynamics. The non-Markovian properties of the TLIB are reflected in
PACS numbers: 78.67.Hc, 63.20.Kr, 63.22.+m, 78.47.+p

many unusual dynamical features such as a nonmonotonic temperature dependence of the initial decay which has also been observed experimentally [10] or a phonon-induced renormalization of the Rabi frequency $[9,14]$. Such features cannot be obtained with models relying on Markovian damping rates often used for other damping mechanisms $[5,6]$. The phase sensitivity inherent in a nonMarkovian type of decoherence also opens new perspectives for an external coherent control of the dephasing $[15,16]$ and may be used to improve general optimal control strategies [17].

Despite the simplicity of the electronic level structure, complete solutions for the dynamics are only available in rare cases like, e.g., the excitation by ultrafast pulse sequences [8]. The more general situation of arbitrarily shaped pulses could only be treated numerically usually involving further approximations. Examples are the perturbation analysis [12] or the correlation $[9,15]$ and cumulant [7] expansions. A serious disadvantage of many of these approximations is that they only apply to weak couplings and low temperatures.

A powerful method for studies of quantum dissipative systems is the path integral formalism $[13,18]$. However, numerical calculations of the relevant real time path integrals run into the well-known problem that many paths of nearly equal weight cancel by destructive phase interference. A numerically accurate approach that overcomes this problem has been developed recently $[19,20]$. To go beyond the limits of perturbative expansions, a similar algorithm is used in this letter to calculate the density matrix of a resonantly excited strongly confined QD. The method allows us to explore the system dynamics for any carrierlight or carrier-phonon coupling strength enabling model studies in the highly nonperturbative regime of strong fields and phonon couplings and high temperatures inaccessible up to now. Our results demonstrate a nontrivial genuinely non-Markovian dynamics of optically excited 
QDs. The decay time of the RO turns out to be a nonmonotonic function of the applied field: it has a minimum at some value of the field strength. When looking at the RR signal, this leads to an interesting phenomenon: a highfield reappearance of the RR. This novel phenomenon is a general feature of the TLIB that occurs for all coupling strengths and temperatures and thus may be observed in many quantum dissipative systems.

The TLIB Hamiltonian reads in the rotating wave approximation (using Bohr units)

$$
\begin{aligned}
H= & \Omega_{0}|0\rangle\left\langle 0\left|+\Omega_{x}\right| x\right\rangle\left\langle x\left|+\sum_{q}\left(\gamma_{q}^{*} b_{q}^{+}+\gamma_{q} b_{q}\right)\right| x\right\rangle\langle x| \\
& +\sum_{q} \omega_{q} b_{q}^{+} b_{q}-\left(\boldsymbol{\mu}^{*} \cdot \mathbf{E}^{*}(t)|0\rangle\langle x|+\boldsymbol{\mu} \cdot \mathbf{E}(t)| x\rangle\langle 0|\right),
\end{aligned}
$$

where $|0\rangle$ is the QD ground state representing the unexcited QD and $|x\rangle$ is the QD exciton state. $\Omega_{0}$ and $\Omega_{x}$ denote the corresponding energies. $b_{q}\left(b_{q}^{+}\right)$are annihilation (creation) operators for phonons with momentum $q$ and frequency $\omega_{q}, \boldsymbol{\mu}$ describes the dipole coupling to the laser field $\mathbf{E}(t)$ and $\gamma_{q}$ denotes the exciton-phonon coupling. We assume $\boldsymbol{\mu} \cdot \mathbf{E}(t)=\frac{1}{2} f(t) e^{-i \Omega t}$, where $f(t)$ is a real envelope and $\Omega$ is the laser frequency in resonance with the exciton transition, i.e. $\Omega=\Omega_{x}-\Omega_{0}-\delta \Omega$ with $\delta \Omega$ being the polaronic shift. We consider, as an example, the deformation potential coupling to longitudinal acoustic (LA) phonons, which is the dominant mechanism for pure dephasing in many self-assembled QD systems $[8,10]$.

We are interested in the reduced electronic density matrix $\bar{\rho}(t)=\operatorname{Tr}_{\mathrm{ph}}\left[U(t) \rho^{(0)} U(t)^{-1}\right]$, where the trace is taken over the phonon states and $\rho^{(0)}$ is the initial density matrix. The QD is initially in its ground state while the phonons are in thermal equilibrium with a Bose distribution $N_{q} . U(t)$ is the time evolution operator of the entire system (QD and phonons). The trace over the phonon variables is evaluated explicitly leading to the real time path integral with the Feynman-Vernon action [18,21]. This procedure fully accounts for all deviations of the phonon system from thermal equilibrium which occur as a consequence of the driven dynamics. To perform this path integration numerically we employ a slightly modified version of the augmented density matrix method [19], supplemented with the "on-fly" screening procedure [20]. Here we shall give a short description of the approach; the details will be given elsewhere. The path integral is discretized by introducing time slices $t_{j}$ and the reduced density matrix is represented as a sum over paths defined on these time slices $[18,19]$ with the discrete Feynman-Vernon action [19] and the memory kernel

$$
\begin{aligned}
K\left(t_{n}-t_{j}\right) & =\int_{t_{n-1}}^{t_{n}} d t \int_{t_{j-1}}^{t_{j}} d t^{\prime} \Gamma\left(t-t^{\prime}\right), \quad t_{n}>t_{j} \\
\Gamma(t) & =\sum_{q}\left|\gamma_{q}\right|^{2}\left[\left(1+2 N_{q}\right) \cos \left(\omega_{q} t\right)-i \sin \left(\omega_{q} t\right)\right] .
\end{aligned}
$$

For a QD coupled to a continuum of LA phonons, $K(t)$ rapidly decreases with increasing $t$, as illustrated in Fig. 1. Thus the system exhibits an effective memory length $\tau_{m}$ such that $K\left(t>\tau_{m}\right)=0$ is a good approximation. Because of this finite memory length the augmented density matrix method provides an efficient recursive algorithm to calculate the reduced density matrix $\bar{\rho}$ [19]. Apart from discretization errors one obtains numerically exact results for the full range of model parameters. At higher $T, \operatorname{Re}(K)$ is larger and the methods works even better. No numerical difficulties were encountered but very strong fields require smaller time slices and larger computer memory.

We have applied this procedure to GaAs prototype QDs of spherical shape with radius $r=5 \mathrm{~nm}$. The phonon coupling has been modeled using the deformation potentials as in Ref. [10]; all other material parameters are listed in Ref. [22]. We assume a rectangular driving pulse. For Gaussian shaped pulses the features discussed in this letter are less pronounced but qualitatively similar. For cutoff times beyond $\tau_{m} \approx 5 \mathrm{ps}$ the results are independent of the truncation.

Figure 2 shows the exciton occupation as a function of time in the presence of a field with Rabi frequency $f$ for three values of $f$. We clearly see damped RO approaching the stationary occupation $1 / 2$ at large times. This time dependence is qualitatively similar to results obtained with phenomenological dampings. However, in Fig. 2 the decay time strongly depends on $f$. We have extracted the effective decay time by fitting an exponentially damped oscillation to our numerical solution. The inset in Fig. 2 shows this decay time as a function of $f$ demonstrating a pronounced nonmonotonic field dependence. At smaller fields the decay time decreases with increasing field, reaching its minimum at $f \approx 2 \mathrm{ps}^{-1}$, while at larger fields the decay time starts to rise. Thus, at a given time the magnitude of the oscillations increases with increasing field in this regime.

Looking at the occupation at a fixed time $\tau$ corresponds to the RR scenario, where the final occupation after a pulse

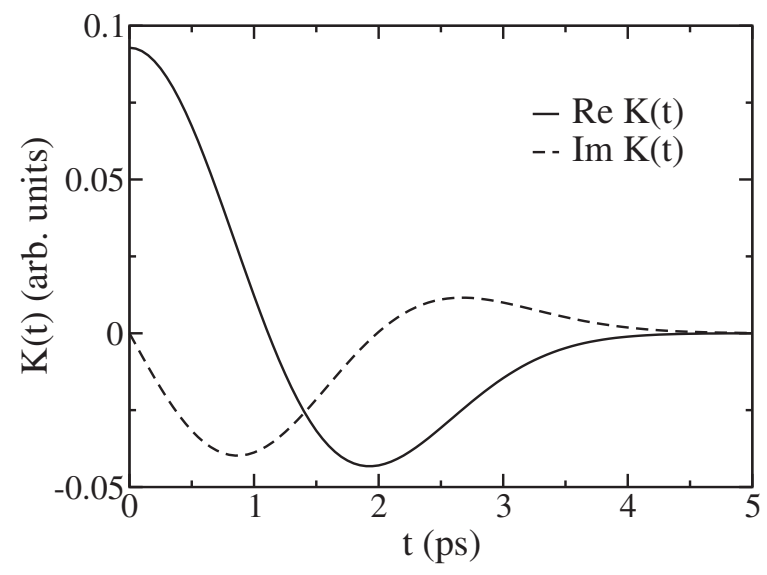

FIG. 1. Time dependence of the memory kernel $K(t)$ for a $5 \mathrm{~nm}$ spherical GaAs QD at temperature $T=10 \mathrm{~K}$. 


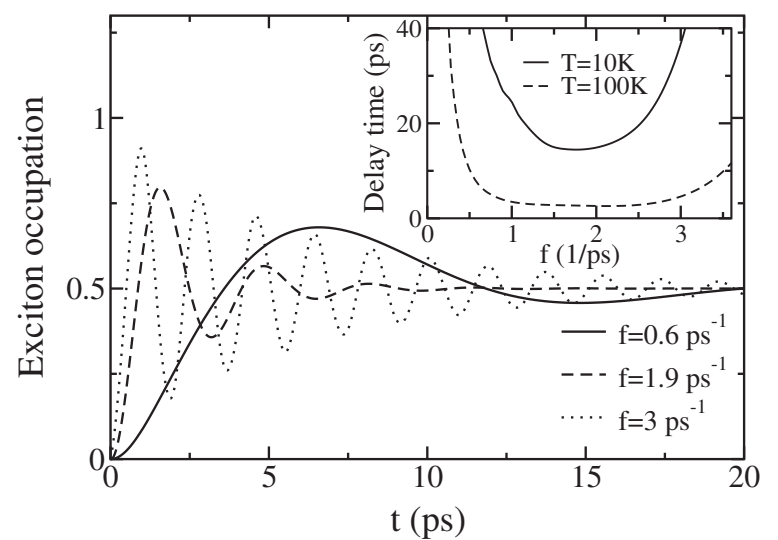

FIG. 2. Time evolution of the QD exciton occupation at $T=$ $100 \mathrm{~K}$ for selected values of the Rabi frequency $f$ exhibiting damped RO. Inset: effective decay time of the RO as a function of $f$ at two temperatures $T$.

is recorded as a function of the PA $f \tau$. Such RR signals are plotted in Fig. 3 for several pulse lengths and temperatures. The period of the rotations evidently depends on $T$ and the PA as discussed previously $[9,14]$. For all pulse lengths the Rabi amplitude first decreases with increasing field, similar to earlier perturbative results [12]. However, with a further increase of the field the oscillations reappear. This reappearance takes place for all pulse lengths and at all temperatures. For shorter pulses it is observed after a smaller number of periods. For $\tau=1 \mathrm{ps}$ the oscillations are almost completely restored after a single period while at $\tau=$ $10 \mathrm{ps}$ the reappearance becomes noticeable only after five periods. The damping of the oscillations is larger at higher $T$ as is intuitively expected. Nevertheless, as seen in Fig. 3 the reappearance takes place at higher $T$ as well but stronger fields are needed.

To explore the strong coupling regime, Fig. 4 shows results of a model study where the exciton-phonon coupling has been increased by hand by factors of 5 and 10 . We clearly see a much more pronounced decay and reappearance which for strong coupling follows an almost complete collapse of the rotations. The coupling $\gamma_{q}$ and the temperature $T$ enter many observables like, e.g., the amplitude of the polarization after a single short pulse, only in the combination [8]: $\left|\gamma_{q}\right|^{2}\left(1+2 N_{q}\right)$, where $T$ is contained in the Bose function $N_{q}$. A higher $T$ essentially implies an effectively increased $\gamma_{q}$ for such observables. Under the present conditions the dependence on $\gamma_{q}$ and $T$ is more involved, but comparing our results in Fig. 4 to a curve obtained for $T=150 \mathrm{~K}$ with the unscaled coupling reveals that the strong coupling limit at low $T$ still yields a decay and reappearance which is similar to the high $T$ behavior of the unscaled model.

It should be noted that the reappearance of RR in Fig. 3 is different from the collapse-and-revival phenomenon of the time-dependent RO in the well-known JaynesCummings (JC) model of a two-level system coupled to a single-mode quantized photon field. Unlike the JC model

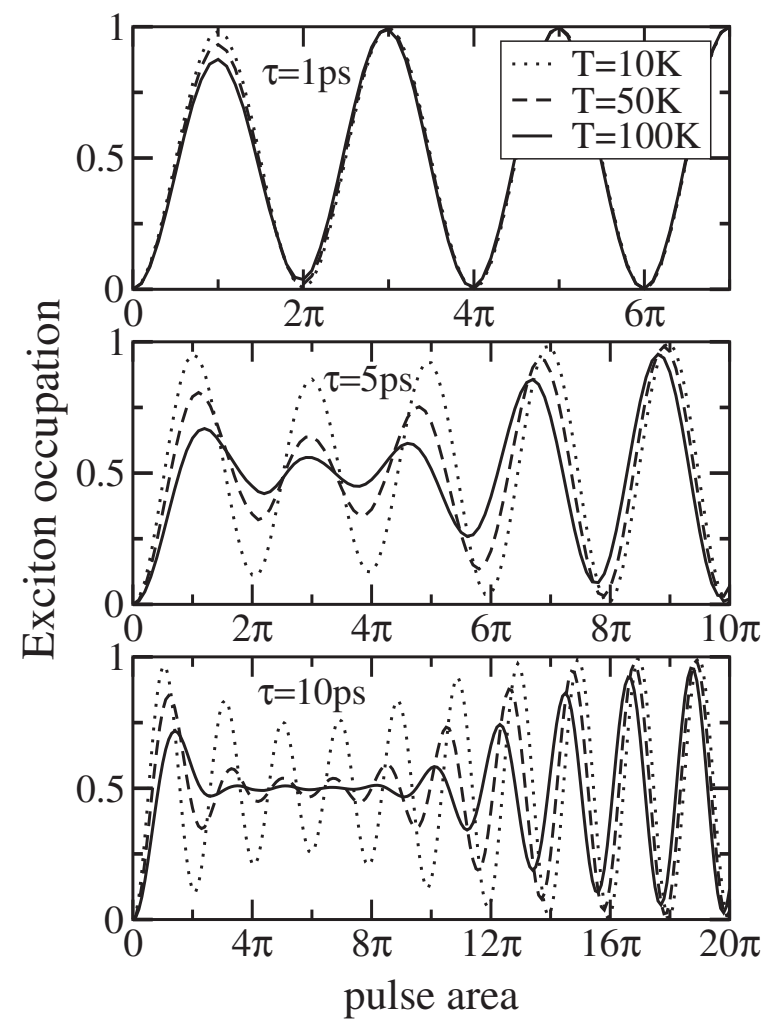

FIG. 3. QD exciton occupation after excitation with a pulse of fixed duration $\tau$ as a function of pulse area calculated at three different temperatures $T$ and durations $\tau$.

which exhibits a periodical collapse-revival of RO in the time-domain, here the RO monotonically decay at arbitrary field strength [see Fig. 2], as expected in a quantum dissipative model with a continuum of phonon modes. Instead we observe the reappearance of RR as a function of the PA. Moreover, the collapse of the oscillations does not repeat periodically. After its reappearance the RR signal does not decay again.

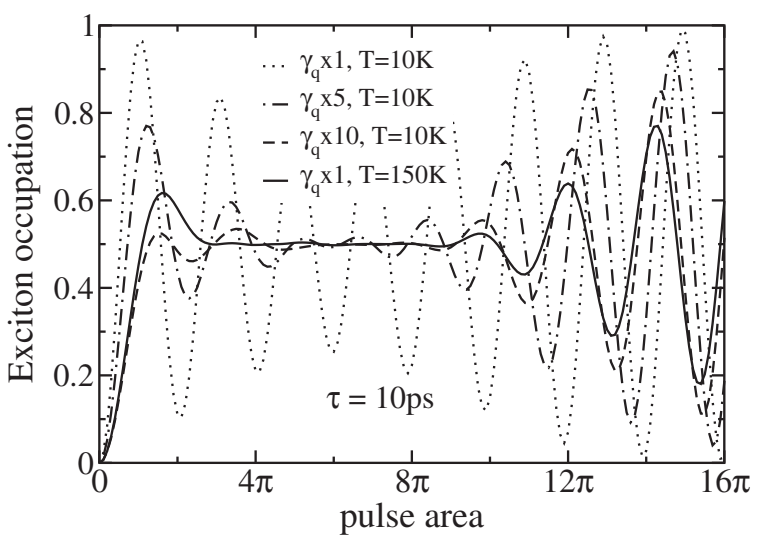

FIG. 4. QD exciton occupation as a function of pulse area at $T=10 \mathrm{~K}$ and $\tau=10 \mathrm{ps}$ for coupling constants $\gamma_{q}$ scaled by factors of 1,5 , and 10. Also shown is the result for the unscaled $\gamma_{q}$ at $T=150 \mathrm{~K}$. 
An understanding of the nonmonotonic damping is achieved by recalling that the phonons can be regarded as harmonic oscillators nonlinearly driven by the electronic dynamics. The damping is dominated by the most strongly coupled phonons which have wavelengths comparable to the QD size [22], in our case corresponding to phonon frequencies $\omega_{\mathrm{ph}} \approx 2 \mathrm{ps}^{-1}$. Obviously, the phonons are most efficiently driven when the driving occurs in resonance with the phonon frequency. It is well known that when the pulses are shorter than $\omega_{\mathrm{ph}}^{-1}$ that RR are undamped $[8,14]$. However, increasing the PA at fixed pulse length speeds up the electronic dynamics. For PAs exceeding $2 \pi$ it is the Rabi period which determines the driving time scale. Thus, even for long pulses, the regime of dynamical decoupling is reached for sufficiently high PAs. Since the damping of the RO is caused by the phonons, the strongest damping is expected for Rabi frequencies $f \approx \omega_{\text {ph }}$, in agreement with our numerical findings. To put this argument on a more quantitative footing we use the result from perturbation theory [12]. The phonon-induced perturbation of the exciton occupation $\delta \rho_{x x}$ is given by [14]

$$
\delta \rho_{x x}=\frac{1}{4} \int_{-\infty}^{\infty} d \omega \frac{R(\omega)}{\omega^{2}} S(\omega),
$$

where $R(\omega)$ is the spectral density of the phonon coupling and $S(\omega)$ comprises the optically controlled dynamics. The explicit form of $S$ in the general case can be found in Ref. [14]. In the case of PA $f \tau=n \pi$, which corresponds to the extrema of the occupation in the phonon-free dynamics, it is given for a rectangular pulse by

$$
S(\omega)=\cos (f \tau) \frac{f^{2}}{4}\left|\frac{e^{i(\omega+f) \tau}-1}{\omega+f}+\frac{e^{i(\omega-f) \tau}-1}{\omega-f}\right|^{2} .
$$

According to Eq. (2) the dephasing is determined by the overlap between $S(\omega)$ and $R(\omega) / \omega^{2}$. The function $R(\omega) / \omega^{2}$ decays exponentially for $\omega>\omega_{\mathrm{ph}}$. The largest peaks of $S(\omega)$ are at $\omega= \pm f$, their width is of the order of $2 \pi / \tau$. Thus, for fixed $\tau$ the overlap in Eq. (2) decreases rapidly when $f$ exceeds $\omega_{\mathrm{ph}}$. Note, $R(\omega)$ broadens with increasing $T$ thus explaining the $T$ dependence in Fig. 3 .

The observability of the reappearance of RR critically depends on two conditions: (i) the electronic dynamics is effectively restricted to a two-level system and (ii) pure dephasing dominates the RR damping. Concerning (i), the maximal damping is reached for Rabi frequencies $f \approx$ $2 \mathrm{ps}^{-1}$ (cf. inset in Fig. 2) corresponding to energies of the order of $1 \mathrm{meV}$. Beyond this value the reappearance of RR sets in. These are rather small values compared to the energy gap between the first and higher excited levels of a strongly confined QD, which is typically larger than several tens of meV. Thus the assumption of an isolated twolevel system is still valid under conditions where we pre- dict the reappearance. Concerning (ii), other dephasing mechanisms can be largely suppressed by choosing suitable samples and experimental set-ups. It has been shown by direct comparison of TLIB calculations with experiments that it is indeed possible to realize a situation where the pure dephasing model yields a quantitative description on a ps time scale [10]. Hence our study should apply to an experimentally reachable parameter range.

To conclude, we discussed the dynamics of a strongly confined QD resonantly excited by external optical pulses. The results were obtained by a numerical evaluation of the density matrix in the path integral representation that is nonperturbative with respect to the phonon-dot and laserdot coupling. For all coupling strengths and temperatures we observe a nonmonotonic field dependence of the RO decay time, which results in a reappearance of the RR at strong fields. This phenomenon can be understood using a rather general resonance argument: the decay ceases when the Rabi frequency exceeds the width of the phonon background spectrum. This suggests that a nonmonotonic field dependence and the related reappearance is a general phenomenon and is expected in a wide range of quantum dissipative systems.

This work is supported by the Belgian Science Policy, the ESF-AQDJJ network, the EU NoE SANDiE and MarieCurie excellence Grant No. NEXT-CT-2005-023778.

[1] E. Biolatti et al., Phys. Rev. Lett. 85, 5647 (2000); F. Troiani et al., Phys. Rev. B 62, R2263 (2000).

[2] T. H. Stievater et al., Phys. Rev. Lett. 87, 133603 (2001).

[3] T. Unold et al., Phys. Rev. Lett. 94, 137404 (2005).

[4] S. Stufler et al., Phys. Rev. B 72, 121301(R) (2005).

[5] Q. Q. Wang et al., Phys. Rev. B 72, 035306 (2005).

[6] J. M. Villas-Bôas et al., Phys. Rev. Lett. 94, 057404 (2005).

[7] E. A. Muljarov et al., Phys. Rev. Lett. 95, 177405 (2005).

[8] A. Vagov et al., Phys. Rev. B 66, 165312 (2002).

[9] J. Förstner et al., Phys. Rev. Lett. 91, 127401 (2003).

[10] A. Vagov et al., Phys. Rev. B 70, 201305(R) (2004).

[11] P. Borri et al., Phys. Rev. B 71, 115328 (2005).

[12] P. Machnikowski et al., Phys. Rev. B 69, 193302 (2004).

[13] U. Weiss, Quantum Dissipative Systems (World Scientific, Singapore, 1999).

[14] A. Krügel et al., Appl. Phys. B 81, 897 (2005).

[15] V. M. Axt et al., Phys. Rev. B 71, 155305 (2005).

[16] U. Hohenester et al., Phys. Rev. Lett. 92, 196801 (2004).

[17] I. Grigorenko et al., Phys. Rev. Lett. 89, 233003 (2002).

[18] A. J. Leggett et al., Rev. Mod. Phys. 59, 1 (1987).

[19] N. Makri and D. Makarov, J. Chem. Phys. 102, 4600 (1995); 102, 4611 (1995).

[20] E. Sim, J. Chem. Phys. 115, 4450 (2001).

[21] R. P. Feynman and F. L. Vernon, Ann. Phys. (N.Y.) 24, 118 (1963).

[22] B. Krummheuer et al., Phys. Rev. B 65, 195313 (2002). 